\title{
National Guidelines for Cognitive Assessment and Rehabilitation of Iranian Traumatic Brain Injury Patients
}

Heshmatollah Ghawami, BSc, MA'; Seyed Behnam Jazayeri, MD²,3; Azad Sabeti Nowsud, MA'; Mahdi Sharif-Alhoseini, MD, PhD ${ }^{3}$; Armin

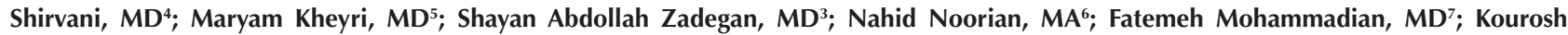
Karimi Yarandi, MD $^{8}$; Akram Azad, PhD ${ }^{9}$; Fariborz Rezaeitalab, $\mathrm{MD}^{10}$; Majid Barekatain, $\mathrm{MD}^{11}$; Jalal Bakhtiyari, PhD ${ }^{12}$; Alireza Moradi, PhD ${ }^{13,14}$; Ahmad Reza Khatoonabadi, PhD ${ }^{15}$; Hojjat Allah Haghgoo, PhD ${ }^{16}$; Mohammad Reza Gheini, MD ${ }^{17}$; Masoud Khadivi, MD ${ }^{18}$; Morteza Faghih Jouibari, MD ${ }^{18}$; Hamid Reza Khayat Kashani, MD ${ }^{19}$; Zahra Mirzaasgari, MD ${ }^{20}$; Ali Meshkini, MD ${ }^{21}$; Hassan Haghshenas, PhD ${ }^{22}$; Fariborz Samini, MD ${ }^{23}$; Parviz Mohit, MD²4; Malahat Akbarfahimi, PhD ${ }^{9}$; Yadollah Alimohammadi, MD ${ }^{25}$; Pooya Payandemehr, MD ${ }^{26}$; Jalal Abbaszadeh Ahranjani, MD²7; Jalil Arabkheradmand, $\mathrm{MD}^{28}$; Zahra Vahabi, MD ${ }^{29}$; Mohammad Arbabi, MD ${ }^{30}$; Hossein Kermanpour, MD ${ }^{31}$; Kavian Ghandehari, MD, FLSP ${ }^{10}$; Nour-Mohammad Bakhshani, PhD ${ }^{32}$; Farhad Motlagh Pirooz, MD ${ }^{33}$; Gholamhossein Ghaedi, MD ${ }^{34}$; Behrooz Dolatshahi, PhD ${ }^{35}$; Seyed Fakhredin Tavakoli, MD $^{33,36}$; Iraj Vosough, MD ${ }^{37}$; Abbas Amirjamshidi, MD ${ }^{8}$; Javad Hatami, PhD ${ }^{38^{*}}$; Vafa Rahimi-Movaghar, $\mathrm{MD}^{3,18,39^{*}}$

${ }^{1}$ Neuropsychology Division, Sina Trauma and Surgery Research Center, Tehran University of Medical Sciences, Tehran, Iran ${ }^{2}$ Students' Scientific Research Center, Tehran University of Medical Sciences, Tehran, Iran

${ }^{3}$ Department of Neurotrauma, Sina Trauma and Surgery Research Center, Tehran University of Medical Sciences, Tehran, Iran ${ }^{4}$ National Agency for Strategic Research in Medical Education (NASR), Tehran, Iran

${ }^{5}$ Clinical Guideline Development and Standardization Group, Office of Health Technology Assessment, Standardization and Tariffs, Deputy for Curative Affairs, Iranian Ministry of Health and Medical Education (MOHME), Tehran, Iran

${ }^{6}$ Department of Psychology, Shahid Rajaee University (SRTTU), Tehran, Iran

${ }^{7}$ Department of Neurology, Roozbeh Hospital, Tehran University of Medical Sciences, Tehran, Iran

${ }^{8}$ Department of Neurosurgery, Sina Hospital, Tehran University of Medical Sciences, Tehran, Iran

${ }^{9}$ Rehabilitation Research Center, Department of Occupational Therapy, School of Rehabilitation Sciences, Iran University of Medical Sciences, Tehran, Iran

${ }^{10}$ Department of Neurology, School of Medicine, Mashhad University of Medical Sciences, Mashhad, Iran

${ }^{11}$ Department of Psychiatry, School of Medicine, Isfahan University of Medical Sciences, Isfahan, Iran

${ }^{12}$ Department of Speech Therapy, Neuromuscular Rehabilitation Research Center, School of Rehabilitation Sciences, Semnan University Medical Sciences, Semnan, Iran

${ }^{13}$ Department of Clinical Psychology, Kharazmi University, Tehran, Iran

${ }^{14}$ Department of Cognitive Psychology, Institute for Cognitive Sciences Studies, Tehran, Iran

${ }^{15}$ Department of Speech Therapy, School of Rehabilitation, Tehran University of Medical Sciences, Tehran, Iran

${ }^{16}$ Department of Occupational Therapy, School of Rehabilitation Sciences, University of Social Welfare and Rehabilitation Sciences, Tehran, Iran

${ }^{17}$ Department of Neurology, Sina Hospital, Tehran University of Medical Sciences, Tehran, Iran

${ }^{18}$ Department of Neurosurgery, Shariati Hospital, Tehran University of Medical Sciences, Tehran, Iran

${ }^{19}$ Department of Neurosurgery, Shahid Beheshti University of Medical Sciences, Tehran, Iran

${ }^{20}$ Neurology Department, Firoozgar Hospital, Iran University of Medical Sciences, Tehran, Iran

${ }^{21}$ Road Traffic Injury Research Center, Department of Neurosurgery, Tabriz University of Medical Sciences, Tabriz, Iran

${ }^{22}$ Department of Psychiatry, Shiraz University of Medical Sciences, Shiraz, Iran

${ }^{23}$ Department of Neurosurgery, Faculty of Medicine, Mashhad University of Medical Sciences, Mashhad, Iran

${ }^{24}$ Department of Neurosurgery, Mazandaran University of Medical Science, Sari, Iran

${ }^{25}$ Neurosurgery Unit, Bahman Hospital, Tehran, Iran

${ }^{26}$ Department of Emergency Medicine, Sina Hospital, Tehran University of Medical Sciences, Tehran, Iran

${ }^{27}$ Department of Neurosurgery, Urmia University of Medical Sciences, Urmia, Iran

${ }^{28}$ Ahya Neuroscience Research Center, Tehran, Iran

${ }^{29}$ Memory and Behavioral Neurology Division, Roozbeh Hospital, Tehran University of Medical Sciences, Tehran, Iran

${ }^{30}$ Department of Psychiatry, Psychosomatic Medicine Research Center, Tehran University of Medical Sciences, Tehran, Iran

${ }^{31}$ Emergency Department, Sina Hospital, Tehran University of Medical Sciences, Tehran, Iran

${ }^{32}$ Department of Psychology, Zahedan University of Medical Sciences, Zahedan, Iran

${ }^{33}$ Department of Neurosurgery, Islamic Azad University, Medical Branch, Mashhad, Iran

${ }^{34}$ Department of Psychiatry, Neurophysiology Research Center, Shahed University, Tehran, Iran

${ }^{35}$ Department of Psychology, School of Behavioral Sciences, University of Social Welfare and Rehabilitation Sciences, Tehran, Iran

${ }^{36}$ Farabi Hospital, Social Security Organization, Mashhad, Iran

${ }^{37}$ Research and Education Department, Razavi Hospital, Mashhad, Iran

${ }^{38}$ Department of Psychology, University of Tehran, Tehran, Iran

${ }^{39}$ Research Centre for Neural Repair, University of Tehran, Tehran, Iran

*Corresponding Authors: Vafa Rahimi-Movaghar, MD; Sina Trauma and Surgery Research Center, Sina General Hospital, Tehran University of Medical Sciences, Hassan Abad Sq., Imam Khomeini St., Tehran, 11365/3876, Iran. Tel: +98-21-66757010; Email: v_rahimi@sina.tums.ac.ir; Javad Hatami, PhD; Department of Psychology, University of Tehran, Tehran, Iran. Tel: +98-21-61117480; Email: hatamijm@ut.ac.ir 


\section{Abstract}

Background: Individuals with moderate to severe traumatic brain injury (TBI) often have prolonged cognitive impairments, resulting in long-term problems with their real-life activities. Given the urgent need for evidence-based recommendations for neuropsychological management of Iranian TBI patients, the current work aimed to adapt eligible international guidelines for cognitive assessment and rehabilitation of the TBI patients in Iran.

Methods: The project was led by an executive committee, under the supervision of the Iranian Ministry of Health and Medical Education (MOHME). Following a systematic literature search and selection process, four guidelines were included for adaptation. Clinical recommendations of the source guidelines were tabulated as possible clinical scenarios for 90 PICO clinical questions covering all relevant phases of care. After summing up the scenarios, our initial list of recommendations was drafted according to the Iranian patients' conditions. The final decision-making, with the contribution of a national interdisciplinary panel of 37 experts from across the country, was conducted in two rounds using online and offline survey forms (Round 1), and face-to-face and telephone meetings (Round 2).

Results: A total of 63 recommendations in six sections were included in the final list of recommendations, among which 24 were considered as key recommendations. In addition, some of the recommendations were identified as fundamental, meaning that proper implementation of the other recommendations is largely dependent on their implementation.

Conclusion: Iranian health policy makers and rehabilitation program managers are recommended to address some fundamental issues to provide the necessary infrastructure to set up an efficient cognitive rehabilitation service system.

Keywords: Cognitive rehabilitation, Guideline, Neuropsychology, Practice guideline, Traumatic brain injury

Cite this article as: Ghawami H, Jazayeri SB, Sabeti Nowsud A, Sharif-Alhoseini M, Shirvani A, Kheyri M, et al. National guidelines for cognitive assessment and rehabilitation of Iranian traumatic brain injury patients. Arch Iran Med. 2020;23(12):813-820. doi: 10.34172/aim.2020.108.

Received: June 23, 2020, Accepted: August 4, 2020, ePublished: December 1, 2020

\section{Introduction}

Traumatic brain injury (TBI) is a traumatic event that results from an external force to the brain, causing disturbances to normal physical, neurologic and cognitive function, and ultimately impacts the patient's physical, psychological and social well-being. ${ }^{1} \mathrm{TBI}$ is a global health challenge, ${ }^{2}$ and a leading cause of death and disability in all countries, with a disproportionate burden in lowincome and middle-income countries (LMICs). ${ }^{3}$ A Global Burden of Disease (GBD) analysis ${ }^{4}$ estimates that there were 27.08 million new cases and 55.50 million prevalent cases of TBI worldwide in 2016, with an increase of $8.4 \%$ in the age-standardized prevalence from 1990 to 2016. The analysis also estimates that 8.1 million years of life lived with disability (YLDs) were caused by TBI in $2016 .{ }^{4}$ Others suggest a larger number for global incidence and prevalence of TBI. For example, Maas et $\mathrm{al}^{3}$ estimate that 50-60 million new cases of TBI will occur each year and about half of the world's population will sustain at least one TBI during their lifetime. Road traffic crashes (RTCs) are the leading cause of TBI in many countries, especially in LMICs. ${ }^{5}$ Iran is among the countries with one of the world's highest rates of RTCs that cause 685611 nonfatal injuries annually. ${ }^{6}$ In a Harvard study on road traffic injuries in 18 countries, ${ }^{7}$ Iran had the highest rate of road injury deaths and over one million Iranians sustained nonfatal injuries due to the RTCs in 2005, with the rate of the injuries peaking in the age group of 15-24 years. A large number of the injured patients experience TBIs and ensuing cognitive complications. According to the GBD study, ${ }^{4}$ in 2016, there were 302610 new cases of and 103,580 YLDs caused by TBI in Iran.

Individuals who have suffered moderate to severe TBIs will often have prolonged impairments in such important cognitive domains as attention, memory, executive abilities, and cognitive aspects of communication. ${ }^{8,9}$ Consequently, the patients will often develop long-term problems with their daily living activities, social integration and occupational function. Cognitive rehabilitation of TBI is "a systematically applied set of medical and therapeutic services designed to improve cognitive functioning and participation in activities that may be affected by difficulties in one or more cognitive domains"(p. 2). ${ }^{10}$ To optimize the outcomes of TBI rehabilitation, the services should be based on up-to-date and high-quality evidence. ${ }^{11}$

In view of the urgent need for evidence-based recommendations for neuropsychological management of Iranian TBI patients in all relevant phases of care, in 2018, the Neuropsychology Division of Sina Trauma and Surgery Research Center (STSRC) at Tehran University of Medical Sciences (TUMS) adapted four eligible international clinical practice guidelines (CPGs) for cognitive assessment and rehabilitation of TBI patients in Iran, with the contribution of a national interdisciplinary panel of experts usually involved in TBI cognitive rehabilitation, from across the country. This report is an abridgment of the project's final report. The project has been conducted under the supervision of the Iranian Ministry of Health and Medical Education (MOHME) and is considered as the national guideline by the Ministry.

\section{Materials and Methods}

The adaptation process was designed and conducted under the technical supervision of Clinical Guideline Development and Standardization Group at MOHME's Deputy for Curative Affairs. The MOHME's "Clinical Practice Guideline Adaptation Model in I.R Iran"12 draws upon international models of CPG adaptation 
including the ADAPTE process, ${ }^{13}$ while considering local conditions. The national model proposes a seven-step process for adapting CPGs in medical practice. The steps are described below with relevant considerations for the rehabilitation context of the current project:

\section{Step 1: Establishing Guideline Adaptation Team}

The project was led by an executive committee. The executive committee consisted of six members including four psychologists, one neurosurgeon, and one general practitioner. The committee members were researchers and/or clinicians with previous experience in cognitive assessment and rehabilitation of TBI patients; two members (S.A.Z and V.R-M) had previously adapted international CPGs for early management of Iranian TBI patients. ${ }^{14}$ All the members received special training in guideline adaption. The executive committee performed steps 1 to 6 , managed the decision-making (step 7), and summed up the results.

Also, a national multidisciplinary panel of experts from all around the country was formed that included 37 relevant specialists involved in TBI care. The expert panel comprised 14 neurosurgeons, six neurologists, five psychologists/neuropsychologists, four psychiatrists/ neuropsychiatrists, three occupational therapists, two speech-language pathologists, two emergency care specialists, and one neuroscientist. The experts were involved in the final decision-making (step 7): making a decision upon the final list of recommendations and the key recommendations, and articulating novel recommendations regarding the infrastructure and fundamental elements necessary for cognitive assessment and rehabilitation of TBI patients in Iran.

\section{Step 2: Specifying Scope and Domain of Guideline}

We decided to adopt the INCOG team's approach ${ }^{8,15}$ when specifying the scope and purpose of our study. The study aimed to offer evidence-based recommendations for the cognitive assessment and rehabilitation of Iranian adults (age $\geq 18$ ) with moderate to severe TBI $($ GCS $<13$ ) in all relevant phases of care. For a comprehensive coverage on key areas of cognitive rehabilitation, we chose to provide the guidelines in six sections: Principles and fundamental recommendations for cognitive assessment and rehabilitation of Iranian TBI patients; Management of Post Traumatic Amnesia (PTA)/Post Traumatic Delirium (PTD); Rehabilitation of attention deficits; Rehabilitation of executive dysfunction; Management of cognitivecommunication disorders; and Rehabilitation of memory impairments.

Behavioral and affective disorders, also deficits usually occurring with focal damages including visuoperceptual and language impairments (which are more relevant for CPGs of strokes than those of TBI that has a diffuse nature), were not covered by the current guidelines.
Step 3: Searching and Retrieving Guidelines

A detailed search for relevant published guidelines was conducted in September, 2018. Twenty websites of guideline clearinghouses and relevant organizations (list of the guideline portals in Table S1), and bibliographic databases including PubMed, PsycINFO, Cochrane Library, and EBSCOhost were searched for records of the last ten years (from January 2008 to September 2018). Also, the first 50 websites were screened for the search terms in Google. The search terms were (a) TBI, Head trauma, Brain injury, or Head injury; AND (b) Guidelines, or recommendations; AND (c) rehabilitation, intervention, practice, or management. Cognitive keywords (cognition, attention, etc.) were not used at this point, because there are several guidelines that have recommendations for cognitive rehabilitation in sections within them, not necessarily represented in the title of the guideline.

Titles, abstracts, and full-texts of identified publications were evaluated using the following inclusion criteria adopted from the INCOG team:

I. Patient group: Only guidelines for adults with moderate to severe TBI were included. Guidelines for patients with mild TBI were excluded.

II. Study Type: CPGs are defined as "systematically developed statements to assist practitioners' and patients' decisions about appropriate healthcare for specific clinical circumstances"16(p38), and referred to henceforth as 'guideline'. For inclusion in this study, each guideline (published in the form of the comprehensive document and/or journal reports) had to include specific evidencebased recommendations, meeting the following two criteria: (a) A systematic approach was used for searching the evidence, and (b) A clear relationship exists between the recommendations and the supporting evidence.

III. Scope: The guideline scope had to exclusively focus on or include a section devoted to cognitive rehabilitation after TBI. Cognitive rehabilitation was defined as treatments that target at least one of the following cognitive functions: attention, executive function, cognitive communication, or memory. The associated guidelines for other topics (e.g., affective disorders, behavioral disorders, perception and language disorders) were excluded.

IV. Language: Only English.

V. The availability of the full text of the guidelines was also necessary for inclusion.

Step 4: Guideline Selection

After screening the retrieved guidelines using the inclusion criteria, the initial selected guidelines were assessed for quality, currency and content. In the final guideline evaluation, four appraisers scored the initial guidelines 
using the Appraisal of Guidelines for Research and Evaluation II (AGREE II). ${ }^{17}$ A cut-off score of $60 \%$ on the key AGREE domain Rigor of Development was used as minimum score on the AGREE II instrument for final guideline selection. In addition, the currency of the literature search of the guidelines, explicit link between the recommendations and their supporting evidence, and applicability and acceptability of the recommendations to the context of use were items used for guideline selection.

\section{Step 5: Developing Clinical Scenarios}

After selecting the final guidelines, clinical recommendations of the guidelines were extracted and tabulated as possible clinical scenarios answering the same PICO (Patient and/or problem; Intervention, or diagnostic test, etc.; Comparison intervention, if relevant; and Outcome) clinical questions. ${ }^{18}$ Recommendations that answered a single question were formulated in a single question and recommendations that included different interventions or populations were formulated as separate questions.

\section{Step 6: Clinical Scenario Evaluation}

At this stage, the clinical scenarios (the recommendations of the final source guidelines) for each PICO question were reviewed for the presence or absence of discrepancy, and since there was no disagreement between the source guidelines on almost all the questions, according to the MOHME's Clinical Guideline Development and Standardization Group, there was no need to analyze different scenarios and decision-making for scenario selection. Therefore, we decided to draft our recommendations by summing up the scenarios from the source guidelines, and adapting them in accordance with the Iranian patients' conditions. We also decided to develop novel recommendations regarding the infrastructure and fundamental elements necessary for cognitive assessment and rehabilitation of the Iranian TBI patients, with the contribution of the national panel of experts.

\section{Step 7: Decision-making}

The final decision-making stage was conducted in two rounds. In the first round, in order to gather the opinion of the national expert panel, a form including the initial list of recommendations was designed in online (using Google Form) and offline (using MS Word software) formats. After being invited and familiarized with the project, the panel members received the forms via email (the full texts of all the source guidelines and a summary of the process were also sent to the members). They were able to add multiplechoice and descriptive answers in both formats. Each recommendation and the level of supporting evidence was presented on one page of the forms, along with questions about adoptability of the recommendation and descriptive suggestions for establishing the infrastructure, where deemed necessary, to implement the recommendations. The questions on adoptability were incorporated as a short form of the "Adoptability" questionnaire presented in the MOHME's adaptation model ${ }^{12}$. The questionnaire assesses three factors: feasibility, generalizability of the effectiveness to the Iranian population, and acceptability by Iranian patients. In addition to the adoptability questions and those about infrastructure suggestions, two additional questions were asked for each recommendation: the first evaluated the eligibility of recommendation to be in the final list of guideline recommendations and the second regarded the list of "key" recommendations. The first round of the expert opinion gathering was conducted from November to December 2018. The process of decisionmaking is summarized in Figure 1. At the beginning, the recommendations that received an Overall Adoptability score of $50 \%$ or less were excluded. For the remaining recommendations, those that had less than $70 \%$ of panel members' votes for being in the final recommendation list were also discarded and those with $85 \%$ or more of the panel votes for inclusion were selected for the final list. Recommendations that received an Overall Adoptability score of more than $50 \%$ and $70-85 \%$ of the panel members voted them to be included in the final list were entered into the second round for further evaluation. In the second round, face-to-face and telephone meetings were held with those panel members who had special expertise and/or relevant research experience in the particular area covered by each recommendation, for more discussion and reaching a consensus. We also conducted an updated literature search of databases and websites for new evidence published since the search date of the source guidelines.

\section{Results}

A total of 1647 records from guideline database websites and 1005 records from bibliographic databases were identified (Figure 2). After excluding 68 duplicates, titles and abstracts of 2584 records were screened by two members of the executive committee, independently. After exclusion of 2525 irrelevant records, the full-texts of 59 guidelines were reviewed and assessed using the inclusion criteria. This process limited the numbers to seven guidelines of which three were excluded after AGREE II evaluation. In the final stage of guideline selection process, the following four guidelines were selected as source guidelines for data extraction, based on the final inclusion criteria (the quality score for the AGREE's Rigor of Development greater than 60/100, the currency of the literature search, etc.):

1. INCOG Guidelines for Cognitive Rehabilitation Following TBI (INCOG team ${ }^{15,19-23}$ ).

2. The INESSS-ONF Clinical Practice Guidelines for the Rehabilitation of Adults with Moderate to Severe $\mathrm{TBI}^{24-25}$ 
A

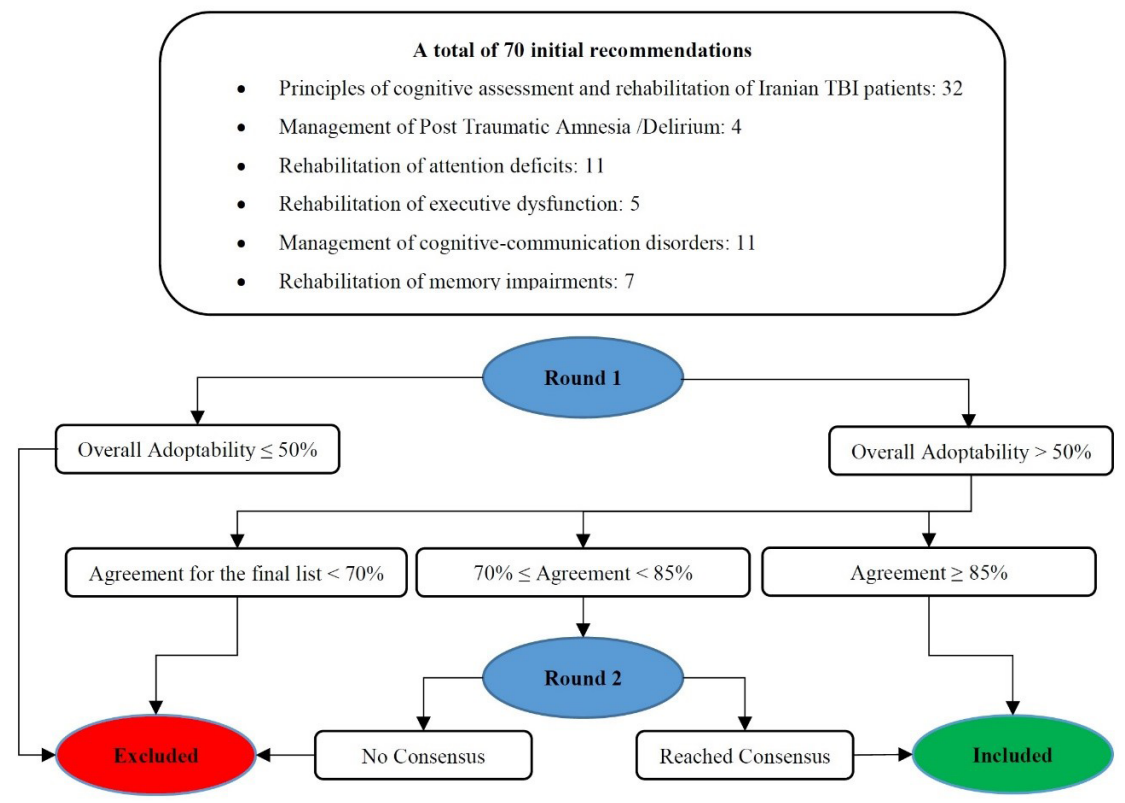

Figure 1. Recommendation Development (A) and Inclusion Process (B)

3. The SIGN's Brain Injury Rehabilitation in Adults: A National Clinical Guideline ${ }^{26}$

4. The ACRM's Evidence-Based Cognitive Rehabilitation: Updated Review of the Literature from 2003 through 2008. ${ }^{27}$ (the extractions were later updated based on the latest update of the review ${ }^{28}$ )
The source guidelines' recommendations were tabulated as possible scenarios for $90 \mathrm{PICO}$ clinical questions covering the six sections related to all stages of recovery. After summing up the scenarios, 70 initial recommendations were drafted according to the Iranian patients' conditions. Based on the expert panel opinions and adapting process,

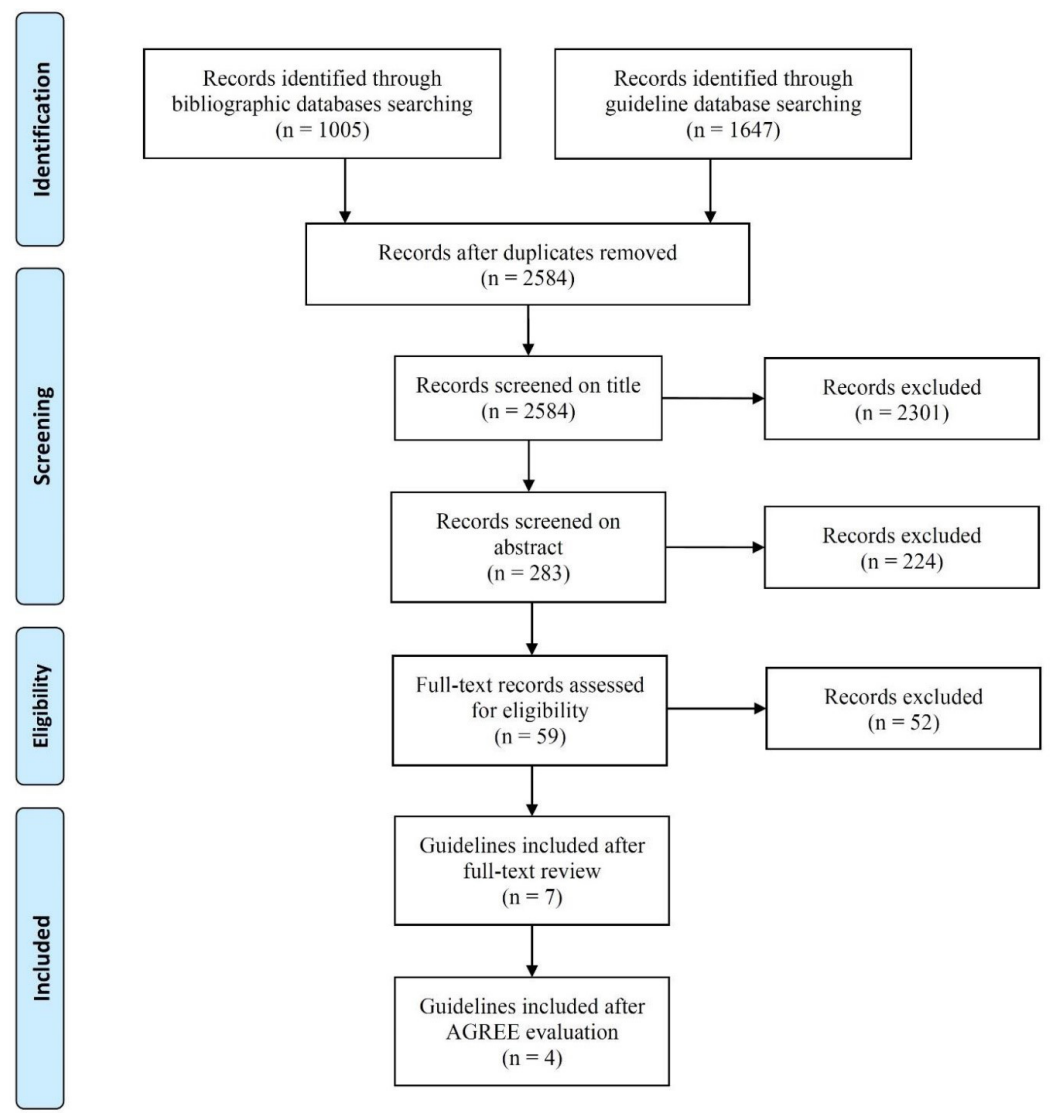

Figure 2. PRISMA Flow Diagram of the Guideline Selection Process. 
61 recommendations were selected. In addition, two new recommendations were developed by summarizing the descriptive opinions of the expert panel members to ensure the infrastructure needs for implementation process. Finally, a total of 63 recommendations were included in the final list of recommendations of the guidelines. Among these recommendations, 24 were considered as key recommendations by the executive team according to the level of evidence and the panel members' votes (at least $60 \%$ of the panel members' votes were necessary for a recommendation to be included in the Guideline Key Recommendations List). In addition, some of the final recommendations were identified as fundamental, meaning that the proper implementation of the rest of the guideline recommendations is largely dependent on their implementation.

\section{Discussion}

To the best of our knowledge, this study is the first attempt to provide evidence-based recommendations for cognitive rehabilitation of persons with moderate to severe TBI in all relevant phases of care based on the cultural and infrastructural context of Iran. After a systematic selection process, four guidelines were included in this study, published by four professional groups/organizations in high-income countries. The INCOG Guideline is one of the most comprehensive published guidelines for cognitive rehabilitation of moderate to severe TBI, developed by an international team of researchers and clinicians. The team's reports and the recommendations have been published in the form of eight articles in the Journal of Head Trauma

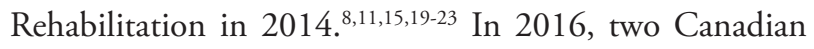
accredited organizations "Institut national d'excellence en santé et en services sociaux" (INESSS) and "Ontario Neurotrauma Foundation” (ONF) released a guideline. The INESSS-ONF guideline, entitled "Clinical Practice Guideline for the Rehabilitation of Adults with Moderate to Severe TBI", ${ }^{24}$ is the most comprehensive and up-todate rehabilitation guideline for these patients. It contains 266 recommendations for various clinical issues related to the rehabilitation of moderate to severe TBI. The guideline of Scottish Intercollegiate Guidelines Network (SIGN), entitled "Brain Injury Rehabilitation in Adults: A National Clinical Guideline", ${ }^{26}$ provides information on post-acute assessment for adults with brain injuries and interventions for cognitive, communicative, emotional, behavioral and physical rehabilitation. For our project, we utilized the relevant recommendations of this guideline for cognitive rehabilitation of TBI. The Cognitive Rehabilitation Task Force (CRTF) of the American Congress of Rehabilitation Medicine (ACRM), Brain Injury Special Interest Group was one of the first teams to undertake a systematic review of evidence for the development of guidelines on "evidence-based cognitive rehabilitation" for brain injury patients including TBI and Stroke. The first report of the CRTF's review, along with evidence-based clinical recommendations, was published in $2000,{ }^{29}$ and subsequent updates were published in $2005^{30}$ and $2011 .^{27}$ Since the official report of the CRTF's last review ${ }^{28}$ was not yet published at the time of our review, we contacted the co-chair of the task force via email and received the clinical recommendations based on the updated review of the literature from 2009 through 2014 (K. Cicerone, personal communication, October 11, 2018). Therefore, when drafting our recommendations, the extractions from the ACRM guideline have been updated according to the CRTF's last review.

The infrastructure necessary for rehabilitation programs is not well established within the healthcare systems of Iran. Thus, when finalizing the recommendations, we identified the need to address some fundamental issues to enable proper implementation of the guideline. With a similar concern, the INESSS-ONF guideline development team have presented 11 "fundamental" recommendations, identified as the elements that must be put in place by program managers in order to build the rest of the system properly. ${ }^{24,25}$ Basic infrastructure requirements and recommendations identified in our study were as follows:

- Educating the acute care staff and patient caregivers to properly deal with the patient according to relevant neuropsychological guidelines (for example, for patients with behavioral symptoms, such as agitation, and amnesia);

- Training at least one of the involved nurses/physicians or recruiting a psychometrician for initial cognitive assessments, including cognitive screening and the serial assessment of PTA, in neurosurgery wards during the acute care (Note: more specialized assessments should be handled by relevant qualified experts);

- Educating and training specialized staff in neuropsychological rehabilitation by adding new topics in the curriculum of related fields as well as establishing formal courses in clinical neuropsychology in universities of the country (for recommended courses for the curriculum of these programs, see Ghawami and Rahimi-Movaghar ${ }^{31}$ );

- Allocating an appropriate room in neurosurgery wards for neuropsychological assessment of the patients before discharge;

- Establishment of specialized rehabilitation clinics within hospitals or referral centers to perform specialized cognitive rehabilitation assessments and interventions recommended in these guidelines after discharging patients from neurosurgery departments. These clinics should have a holistic, multidisciplinary approach, with appropriate rehabilitation programs, tools and equipment, as well as necessary specialized personnel (rehabilitation specialists such as psychologists, neurologists, neuropsychiatrists, occupational therapists, speech-language pathologists, 
social workers, etc.). The rehabilitation assessments and interventions should focus on the principal goal of "improving the patient's performance in real life."

- Attempting to get insurance coverage for the patients' cognitive rehabilitation services;

- Investment in developing, translating, and cultural validating of cognitive rehabilitation protocols and tools, recommended in the guidelines.

In addition to the need for the above executive actions to provide the necessary infrastructure, rehabilitation program managers and their leaders need to prioritize the following basic steps to set up an efficient cognitive rehabilitation service system:

- Establishing a mechanism for gathering sufficient information for all conscious TBI patients in relation to common physiological and psychological disorders that interfere with the cognitive rehabilitation process (Recommendation 1-2);

- Monitoring the target population of the rehabilitation services by collecting and analyzing data on clinical and socio-demographic profile of the patients (Recommendation 1-14);

- Monitoring key aspects of the processes and efficacy of the TBI rehabilitation programs (Recommendation 1-15);

- Establishing a multidisciplinary rehabilitation team whose membership is based on the TBI patient's developing needs as identified by initial and ongoing assessments and goal-setting with the patient and his/ her family (Recommendation 1-17).

The current guidelines provide a relatively comprehensive coverage on key areas of cognitive rehabilitation in all relevant phases of recovery for the Iranian TBI patients. We attempted to include representatives of all specialties involved in cognitive assessment and rehabilitation of the TBI patients. One strength of this work was our success in communicating and collaborating with experts all over the country. The response rate in the first and second rounds of online surveys were $88 \%$ and $100 \%$ respectively, which is higher than average response rates in organizational surveys $(\mathrm{M}=52.7 \%, \mathrm{SD}=20.4 \%) .{ }^{32}$ This indicates a successful survey design and follow-up method in this project. At first, three members of the executive committee (H.G, A.S, and N.N) were responsible for contacting all the expert panel members via phone call. Then, the emails containing the forms were sent to the panel, and were followed up by the executive members. The email and the survey form were prepared in a convenient and userfriendly format, tailored to the respondents' limitations in time and internet speed across the country. For example, given that in some provinces of the country the internet speed is low and therefore loading and filling out a 70-page online form takes a large amount of time, we decided to give the other option of responding to the form in offline format (downloading and completing an interactive MS
Word form). The forms would take about two hours from each expert and they had up to one week to respond. After one week, the responses were reviewed and those who did not respond were called again.

The current study had some limitations. We did not have relevant experts of health policy and cost-effectiveness analysis in our team. Indeed, the evidence for or against the cost-effectiveness of cognitive rehabilitation is very limited. ${ }^{15}$ Another limitation was that we did not include the patients' and insurance agents' opinions. We know that without considering the local contexts and individual profiles of TBI patients and their preferences, certain interventions recommended in evidence-based guidelines may be infeasible. We recommend considering these issues when implementing these guidelines.

\section{Authors' Contribution}

HG, ASh, MKhe, VR-M and JH: Study concept, design and supervision. HG, ASN and SBJ: Litrature review. HG, ASN, NN and SAZ: Data collection and analysis. HG and SBJ: Drafting of the manuscript. HG, ASN, MS-A, VR-M and JH: Editing of the manuscript. MS-A, FMo, KKY, AAz, FR, MB, JB, AMo, ARK, HAH, MRG, MKha, MFJ, HRKK, ZM, AMe, HH, FS, PM, MAk, YA, PP, JAA, JA, ZV, MAr, HK, KG, N-MB, FMP, GG, BD, SFT, IV, AAm, JH, VR$M$ : Involved in final decision-making (recommendation review and revision, and articulating novel recommendations) of the project and critical revision of the manuscript for important intellectual content.

\section{Conflict of Interest Disclosures}

The authors declare that they have no conflict of interest.

Ethical Statement

Not applicable.

\section{Acknowledgements}

This research was supported by Cognitive Science and Technologies Council (CSTC), Iran's Vice-Presidency for Science and Technology. The project was conducted in the Neuropsychology Division, Sina Trauma and Surgery Research Center (STSRC), Tehran University of Medical Sciences (TUMS). The methodology was designed and supervised by Clinical Guideline Development and Standardization Group; Office of Health Technology Assessment, Standardization and Tariffs; Deputy for Curative Affairs; Iranian Ministry of Health and Medical Education (MOHME). The final report of the project has been approved by the council. We would like to thank Dr. Keith Cicerone and Dr. Mark Bayley for their helpful information.

\section{Supplementary Materials}

Supplementary file 1 contains Table S1.

\section{References}

1. Khan F, Baguley IJ, Cameron ID. 4: rehabilitation after traumatic brain injury. Med J Aust. 2003;178(6):290-5. doi: 10.5694/ j.1326-5377.2003.tb05199.x.

2. Johnson WD, Griswold DP. Traumatic brain injury: A global challenge. Lancet Neurol. 2017;16(12):949-50. doi: 10.1016/ S1474-4422(17)30362-9.

3. Maas AIR, Menon DK, Adelson PD, Andelic N, Bell MJ, Belli A, et al. Traumatic brain injury: integrated approaches to improve prevention, clinical care, and research. Lancet Neurol. 2017;16(12):987-1048. doi: 10.1016/S14744422(17)30371-X.

4. James SL, Theadom A, Ellenbogen RG, Bannick MS, MontjoyVenning W, Lucchesi LR, et al. Global, regional, and national 
burden of traumatic brain injury and spinal cord injury, 19902016: A systematic analysis for the Global Burden of Disease Study 2016. Lancet Neurol. 2019;18(1):56-87. doi: 10.1016/ S1474-4422(18)30415-0.

5. Alshafai N, Maas AIR. Epidemiology of head injury and outcome after head injury. In: Kirollos R, Helmy A, Thomson S, Hutchinson P, eds. Oxford Textbook of Neurological Surgery. Oxford, UK: Oxford University Press; 2019:475-81.

6. World Health Organization. Global Status Report on Road Safety 2009. Available from: https://www.who.int/violence injury_prevention/road_safety_status/2009/en/.

7. Bhalla K, Sharaz S, Abraham J, Bartels D, Yeh PH. Road Injuries in 18 Countries: Methods, data sources and estimates of the national incidence of road injuries. Boston, MA: Harvard University; 2011.

8. Bragge P, Pattuwage L, Marshall S, Pitt V, Piccenna L, StergiouKita $M$, et al. Quality of guidelines for cognitive rehabilitation following traumatic brain injury. J Head Trauma Rehabil. 2014;29(4):277-89. doi: 10.1097/HTR.0000000000000066.

9. Ghawami H, Sadeghi S, Raghibi M, Rahimi-Movaghar V. Executive Functioning of Complicated-Mild to Moderate Traumatic Brain Injury Patients with Frontal Contusions. Appl Neuropsychol Adult. 2017;24(4):299-307. doi: 10.1080/23279095.2016.1157078.

10. Katz DI, Ashley MJ, O'Shanick GJ, Connors SH. Cognitive rehabilitation: the evidence, funding and case for advocacy in brain injury. McLean, VA: Brain Injury Association of America; 2006.

11. Bayley MT, Teasell RW, Wolfe DL, Gruen RL, Eng JJ, Ghajar J, et al. Where to build the bridge between evidence and practice?: Results of an international workshop to prioritize knowledge translation activities in traumatic brain injury care. J Head Trauma Rehabil. 2014;29(4):268-76. doi: 10.1097/ HTR.0000000000000053.

12. Yazdani S, Shirvani A, Nejati M, Heydarpour P, Ahmadi A. Clinical Practice Guideline Adaptation Model in I.R Iran. Iranian Ministry of Health and Medical Education; 2015.

13. Guidelines International Network. ADAPTE Resource Toolkit for Guideline Adaptation Version 2.0; 2009. Available from: https://g-i-n.net/document-store/working-groups-documents/ adaptation/resources/adapte-resource-toolkit-guidelineadaptation-2-0.pdf/view.

14. Abdollah Zadegan S, Ghodsi SM, Arabkheradmand, J, Amirjamshidi A, Sheikhrezaei A, Khadivi M, et al. Adaptation of traumatic brain injury guidelines in Iran. Trauma Mon. 2016;21(2):e28012. doi: 10.5812/traumamon.28012.

15. Bayley MT, Tate R, Douglas JM, Turkstra LS, Ponsford J, StergiouKita M, et al. On behalf of the INCOG Expert Panel. INCOG guidelines for cognitive rehabilitation following traumatic brain injury: methods and overview. J Head Trauma Rehabil. 2014;29(4): 290-306. doi: 10.1097/HTR.0000000000000070.

16. Field MJ, Lohr KN. Clinical Practice Guidelines: Directions for a New Program. Washington, DC: National Academy Press; 1990.

17. Brouwers MC, Kho ME, Browman GP, Burgers JS, Cluzeau F, Feder G, et al. AGREE II: Advancing guideline development, reporting and evaluation in healthcare. Can Med Assoc J. 2010;182(18)e839-842. doi: 10.1503/cmaj.090449.

18. Bragge P. Asking good clinical research questions and choosing the right study design. Injury. 2010;41(Suppl 1):S3-6. doi: 10.1016/j.injury.2010.04.016.

19. Ponsford J, Janzen S, McIntyre A, Bayley M, Velikonja D, Tate R. INCOG recommendations for management of cognition following traumatic brain injury, part I: posttraumatic amnesia/ delirium. J Head Trauma Rehabil. 2014;29(4):307-20. doi:10.1097/HTR.0000000000000074.

20. Ponsford J, Bayley M, Wiseman-Hakes C, Togher L, Velikonja D, McIntyre A, et al. INCOG recommendations for management of cognition following traumatic brain injury, part II: attention and information processing speed. J Head Trauma Rehabil. 2014;29(4):321-37. doi: 10.1097/HTR.0000000000000072.

21. Tate R, Kennedy M, Ponsford J, Douglas J, Velikonja D, Bayley M, et al. INCOG recommendations for management of cognition following traumatic brain injury, part III: executive function and self-awareness. J Head Trauma Rehabil. 2014;29(4):33852. doi: 10.1097/HTR.0000000000000068.

22. Togher L, Wiseman-Hakes C, Douglas J, Stergiou-Kita M, Ponsford J, Teasell R, et al. INCOG recommendations for management of cognition following traumatic brain injury, part IV: cognitive communication. J Head Trauma Rehabil. 2014;29(4):353-68. doi: 10.1097/HTR.0000000000000071.

23. Velikonja D, Tate R, Ponsford J, McIntyre A, Janzen S, Bayley M. INCOG recommendations for management of cognition following traumatic brain injury, part V: memory. J Head Trauma Rehabil. 2014;29(4):369-86. doi: 10.1097/ HTR.0000000000000069.

24. INESSS \& ONF. Clinical Practice Guideline for the Rehabilitation of Adults with Moderate to Severe TBI. 2016. Available from: https://braininjuryguidelines.org/modtosevere/.

25. Bayley MT, Lamontagne M-E, Kua A, Marshall S, MarierDeschenes P, Allaire A-S, et al. Unique Features of the INESSS-ONF Rehabilitation Guidelines for Moderate to Severe Traumatic Brain Injury: Responding to Users' Needs. J Head Trauma Rehabil. 2018;33(5):296-305. doi: 10.1097/ HTR.0000000000000428.

26. Scottish Intercollegiate Guidelines Network (SIGN). Brain injury rehabilitation in adults: a national clinical guideline (SIGN publication no. 130). Edinburgh: SIGN; 2013. Available from: https://www.sign.ac.uk/media/1068/sign130.pdf.

27. Cicerone KD, Langenbahn DM, Braden C, Malec JF, Kalmar $\mathrm{K}$, Fraas $\mathrm{M}$, et al. Evidence-based cognitive rehabilitation: Updated review of the literature from 2003 through 2008. Arch Phys Med Rehabil. 2011;92(4):519-30. doi: 10.1016/j. apmr.2010.11.015.

28. Cicerone KD, Goldin Y, Ganci K, Rosenbaum A, Wethe JV, Langenbahn DM, et al. Evidence-based cognitive rehabilitation: Systematic review of the literature from 2009 through 2014. Arch Phys Med Rehabil. 2019;100(8):1515-33. doi: 10.1016/j. apmr.2019.02.01.

29. Cicerone KD, Dahlberg C, Kalmar K, Langenbahn DM, Malec JF, Bergquist TF, et al. Evidence-based cognitive rehabilitation: Recommendations for clinical practice. Arch Phys Med Rehabil. 2000;81(12):1596-615. doi: 10.1053/apmr.2000.19240.

30. Cicerone KD, Dahlberg C, Malec JF, Langenbahn DM, Felicetti T, Kneipp S, et al. Evidence-based cognitive rehabilitation: Updated review of the literature from 1998 through 2002. Arch Phys Med Rehabil. 2005;86(8):1681-92. doi: 10.1016/j. apmr.2005.03.024.

31. Ghawami H, Rahimi-Movaghar V. Clinical Neuropsychology and Traumatic Brain Injury Patients in Iran [Persian]. 2015. Available from: https://www.researchgate.net/ publication/286930526_Clinical_Neuropsychology_and_ Traumatic_Brain_Injury_Patients_in_Iran_in_Persian.

32. Baruch $Y$, Holtom BC. Survey response rate levels and trends in organizational research. Hum Relat. 2008;61(8):1139-1160. doi: $10.1177 / 0018726708094863$. 